\title{
Does exposure to analgesics in utero cause schizophrenia?
}

\author{
Richard L O'Reilly MB FRCPC
}

$\mathrm{T}$ he cause of schizophrenia, a serious mental illness characterized by hallucinations, delusions and a marked deterioration in social functioning, is an enigma that continues to perplex the scientific community. While we know that approximately $80 \%$ of the risk of developing schizophrenia is conferred by genes (1), no research group has yet located a gene(s) contributing to the cause of schizophrenia. Moreover, while a significant environmental contribution is indicated by the fact that one-half of monozygotic twins pairs are discordant for schizophrenia, the nature of this environmental contribution is still controversial. The past theory that an abnormal family upbringing was the causative environmental factor has not stood up to scientific scrutiny.

In recent years, the search for causative environmental factors has focused on the possibility that some type of prenatal insult predisposes the fetus to develop schizophrenia in early adult life. The initial suspicion of an intrauterine insult was based on indirect evidence. First, dermatoglyphic patterns in patients with schizophrenia were noted to be abnormal. The dermal ridges are laid down between the third and fifth month of gestation, in effect leaving a fossilized record of neurodevelopmental perturbation. Second, minor physical anomalies, typically involving the mouth, ears and eyes, occur with increased frequency in schizophrenia. Much like dermatoglyphic abnormalities, minor physical anomalies represent a disruption of the final phase of the developmental shaping of the face, which takes place in the second trimester. Of course, both abnormal dermatoglyphic patterns and minor physical anomalies may reflect the effects of abnormal genetic regulation of development rather than an extrinsic insult.

Of interest to the readers of this journal is the recent paper by Sorensen et al (2) that reports direct evidence that an extrinsic insult to the fetus, specifically, ingestion of analgesics by pregnant mothers, may increase a person's risk of developing schizophrenia. The authors obtained a detailed record of the medication ingested during pregnancy from pre- and postnatal interviews with the mothers of the Copenhagen Perinatal Cohort (9125 individuals born between 1959 and 1961). By linking these data with the excellent Danish computerized register of psychiatric admissions, they were able to calculate the relative risk of having an admission for schizophrenia in members of the cohort exposed to analgesics in utero compared with those unexposed to analgesics in utero. Almost all individuals with schizophrenia were admitted to hospital during their illness, so we can be confident that most cases of schizophrenia were correctly identified. Sorensen et al (2) calculated the ORs of developing schizophrenia after analgesic exposure in each of the first three months of pregnancy and for the second and third trimesters. An increased risk of developing schizophrenia was seen only with exposure in the third month of the first trimester and with exposure in the second trimester. The OR for second trimester exposure was 4.22 (95\% CI 2.0 to 8.8).

How are we to interpret these findings? The authors studied the effects of a number of different types of medication on the incidence of schizophrenia and previously published a report (3) indicating that exposure to diuretics in the third trimester was associated with an elevated risk of developing schizophrenia. Are the positive findings simply the result of data trawling and multiple analyses?

There actually is an a priori reason to study fetal exposure to analgesics in schizophrenia. Previous research found an increased incidence of schizophrenia in the offspring of mothers who had influenza during the second trimester $(4,5)$. Sorensen et al questioned whether drugs used to alleviate the symptoms of influenza, such as analgesics, might mediate this increased risk. Their findings gain credibility from the fact that the greatest risk occurs with exposure during gestational months 3 through 6, which is the critical time for developing dermatoglyphic and minor physical anomalies, and the period of risk noted in the influenza studies.

Perhaps the analgesic use is simply a marker for influenza infection in pregnant women. This seems unlikely, because mothers in the cohort were questioned about viral infections and there was no association between maternal reports of viral infections during pregnancy and the development of schizophrenia in the offspring. Furthermore, only 15 cohort members were exposed to both influenza and analgesics in the second trimester and none of their offspring developed schizophrenia.

Could the analgesic use have occurred more frequently in mothers who had or were at risk of developing schizophrenia and, thus, were more likely to convey a genetic liability for the illness to their offspring? Again, this seems unlikely. The authors report that there was no association between maternal psychoactive medication use and the development of schizophrenia in the offspring. The use of psychoactive medications is likely to be a better marker than analgesics of genetic predisposition in the mothers. The authors also ran a second analysis in which they excluded all offspring whose mother or father had a psychiatric admission after 1969, but the fourfold increased risk with maternal analgesic ingestion remained.

So should we prescribe analgesics for pregnant women? A good rule of thumb is to avoid prescribing all but essential drugs during pregnancy. Schizophrenia is a serious and debilitating disease and most mothers would be concerned about

University of Western Ontario and Regional Mental Health Care, London, Ontario.

Correspondence: Dr Richard L O'Reilly, Regional Mental Health Care - London, PO Box 5532, Station B, London, Ontario N6A 4H1 .

Telephone 519-455-5110 ext 47240, fax 519-455-5090, e-mail roreilly@uwo.ca 
ingesting a drug that could potentially quadruple their child's chances of developing this illness. Furthermore, schizophrenia is a common disorder affecting $1 \%$ of the population. A prenatal insult that increases the incidence of a common disorder by a factor of four will affect many more individuals than an insult producing a similar elevation in the risk of developing a rare congenital disorder.

To be confident that there really is an association between maternal analgesic use and schizophrenia, we would need to replicate the study using an independent sample. The problem is that we now use many analgesic drugs that were not available in 1959 to 1961 . We can, with relative ease, establish the risks that newer analgesics and anti-inflammatory drugs will cause either congenital abnormalities or perinatal problems (6). In contrast, to determine if fetal exposure to new analgesics increase the risk of schizophrenia, we need to wait 40 years (to allow the cohort to pass through the age of risk for developing schizophrenia).

The evidence reported by Sorensen's group indicates that it would be prudent to avoid using aspirin and codeine in midpregnancy. Aspirin and codeine were among the most common analgesics ingested by mothers of the Copenhagen Cohort. The authors also reported that of the 18 fetuses exposed to morphine or opium at any stage of pregnancy, two subsequently developed schizophrenia - a 10-fold increase over the expected rate. However, further analysis revealed that many of the mothers took morphine or opium following abdominal surgery and had various complications of pregnancy known to confer an increased risk of schizophrenia to the offspring (7). Some reassurance can be drawn from the fact that there was no evidence for an increased risk of developing schizophrenia when analgesics were used early in gestation before pregnancy is recognized, or late in pregnancy when analgesics are more often needed.

\section{REFERENCES}

1. Sullivan PF, Kendler KS, Neale MC. Schizophrenia as a complex trait: Evidence from a meta-analysis of twin studies. Arch Gen Psychiatry 2003;60:1187-92.

2. Sorensen HJ, Mortensen EL, Reinisch JM, Mednick SA. Association between prenatal exposure to analgesics and risk of schizophrenia. Br J Psychiatry 2004;185:366-71.

3. Sorensen HJ, Mortensen EL, Reinisch JM, Mednick SA. Do hypertension and diuretic treatment in pregnancy increase the risk of schizophrenia in offspring? Am J Psychiatry 2003;160:464-8.

4. Mednick SA, Machon RA, Huttunen MO, Bonett D. Adult schizophrenia following prenatal exposure to an influenza epidemic. Arch Gen Psychiatry 1988;45:189-92.

5. O'Callaghan E, Sham P, Takei N, Glover G, Murray RM. Schizophrenia after prenatal exposure to 1957 A2 influenza epidemic. Lancet 1991;337:1248-50.

6. Ostensen ME, Skomsvoll JF. Anti-inflammatory pharmacotherapy during pregnancy. Expert Opin Pharmacother 2004;5:571-80.

7. Hultman CM, Sparen P, Takei N, Murray RM, Cnattingius S. Prenatal and perinatal risk factors for schizophrenia, affective psychosis, and reactive psychosis of early onset: Case-control study. BMJ 1999;318:421-6. 


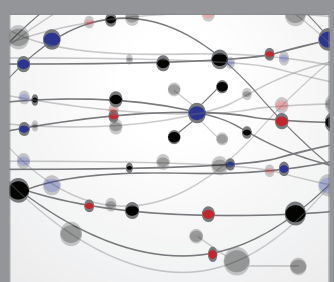

The Scientific World Journal
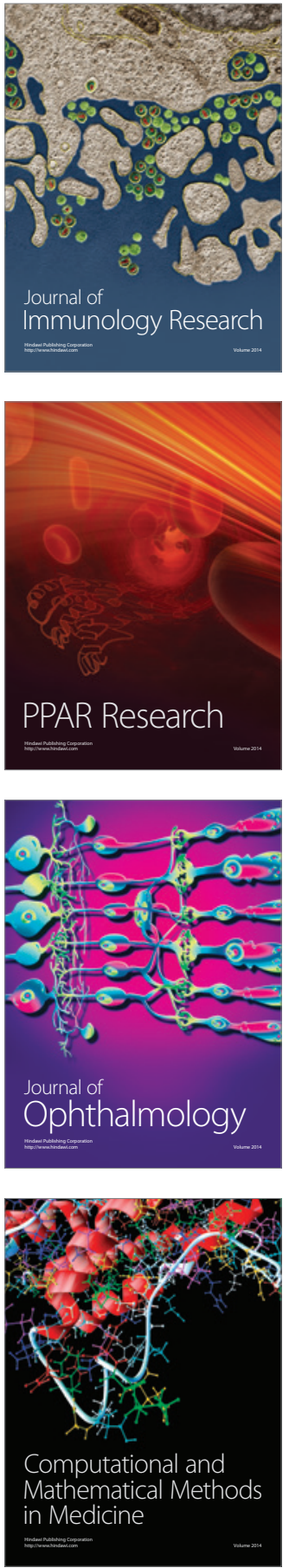

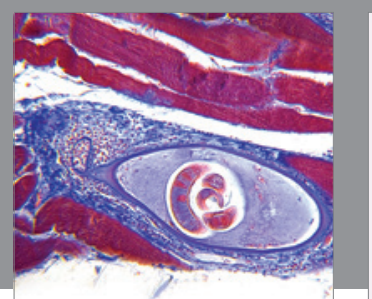

Gastroenterology Research and Practice

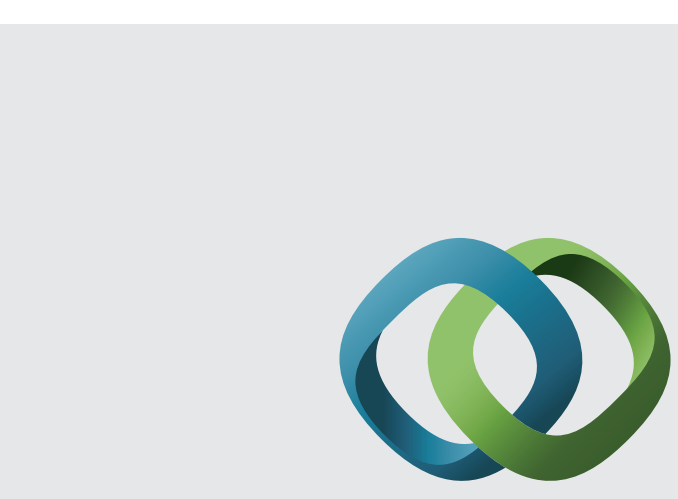

\section{Hindawi}

Submit your manuscripts at

http://www.hindawi.com
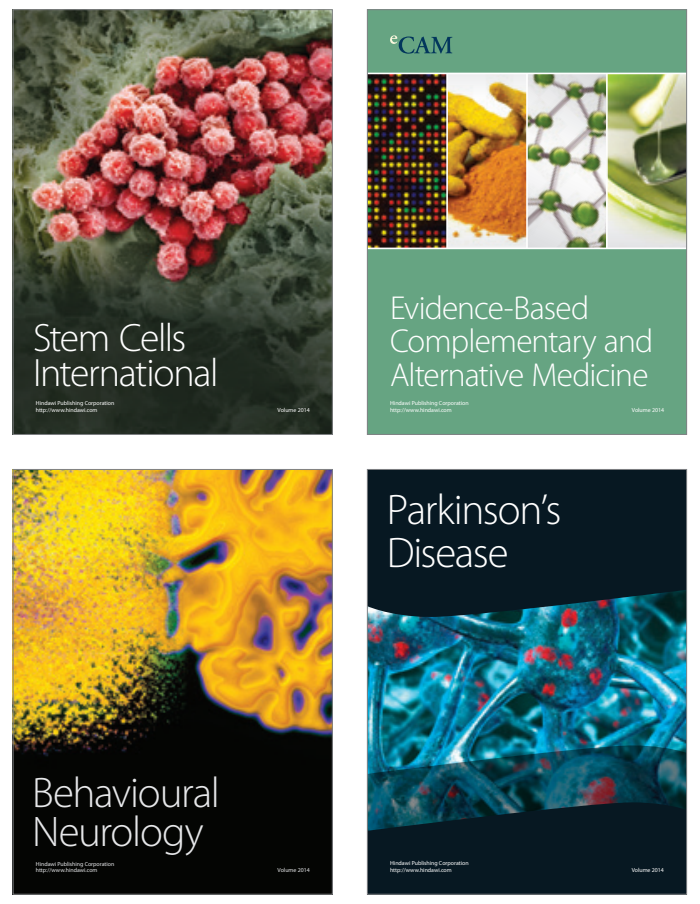
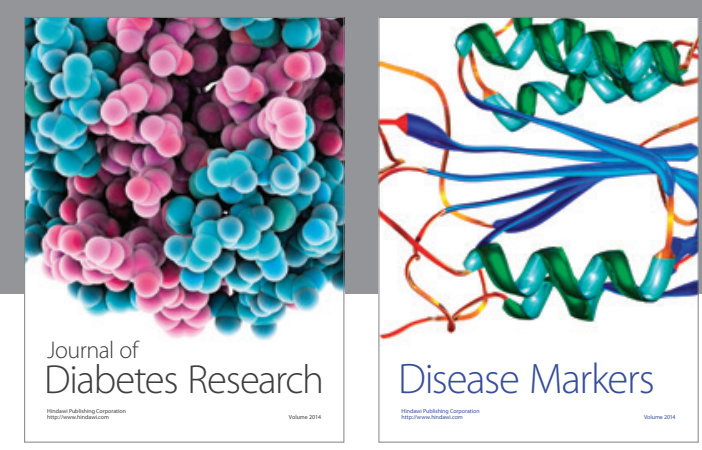

Disease Markers
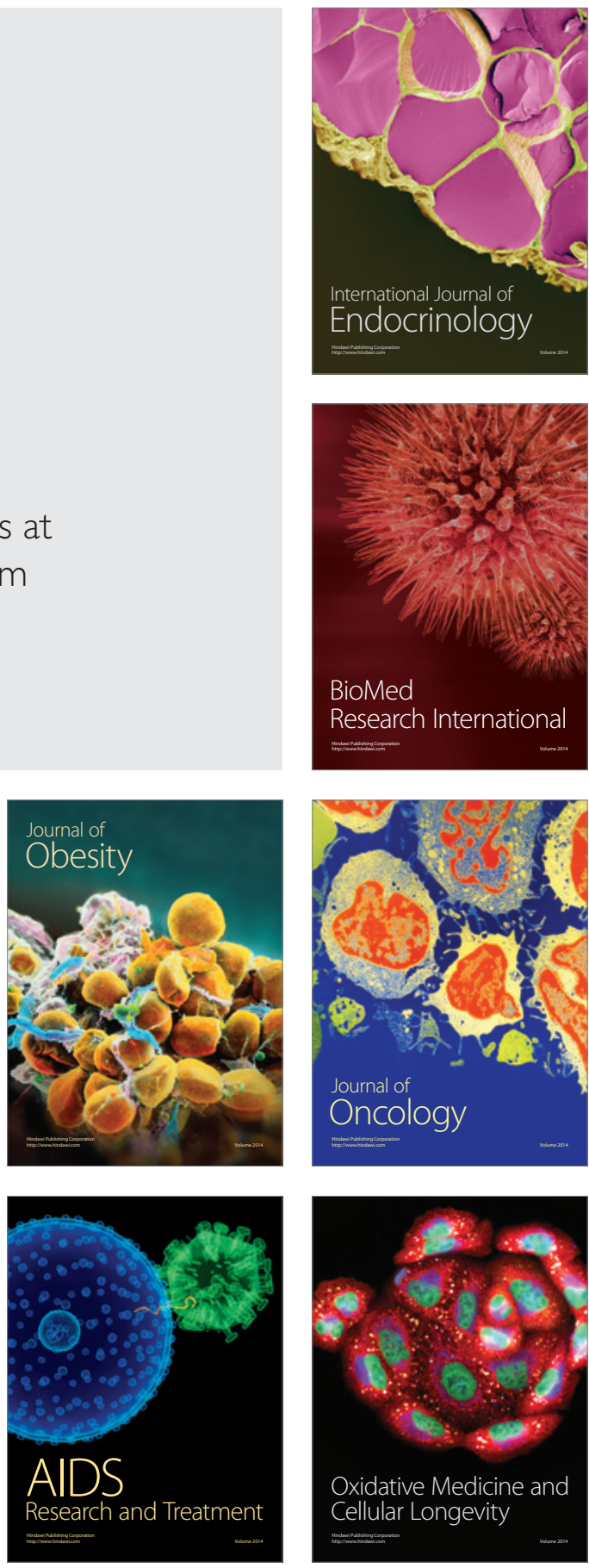